\title{
Effects of microgravity on bone
}

\begin{abstract}
During space missions cosmonauts and astronauts suffer the effects of the absence of gravity. One of the most affected systems is the musculoskeletal with marked loss of bone mass. To know the effects of microgravity is essential for promoting treatments and preventive measures. However, there is a limitation in the collection of data regarding the decrease of bone mass due to the reduced number of space missions. To meet this need, animal models are used. The hind limb unloading method generates loss of bone mass in rodents through the effects of unloading on the hind limbs. In this background, the purpose of this mini review is to briefly present knowledge about the results obtained at the moment with the space flights studies and the use of animal models to clarify the effects of microgravity on bone. In addition, we briefly comment on treatments designed to minimize bone loss in astronauts and cosmonauts.
\end{abstract}

Keywords: microgravity, bone, bone mineral density, osteoporosis
Volume 2 Issue 4 - 2018

\section{Jéssica Suzuki Yamanaka, Marcela Britto de Paiva, Bruna Leonel Carlos, Antônio Carlos Shimano}

Departamento de Biomecânica, Universidade de São Paulo, Brazil

Correspondence: Antônio Carlos Shimano, Departamento de Biomecânica, Medicina e Reabilitação do Aparelho Locomotor, Faculdade de Medicina de Ribeirão Preto, Universidade de São Paulo, Rua Pedreira de Freitas, s/n, 14049-900, Ribeirão PretoSão Paulo, Brazil,Tel +55 1633150225 ,

Email ashimano@fmrp.usp.br

Received: June 14, 2017 | Published: August 02, 2018

\section{Introduction}

The human body is a complex system control by mechanical forces Unbalance of these forces should cause different effects on the health. When a human is submitted to extreme altered conditions the body might have unfavorable responses. In the case of space man there are many problems related theirs conditions of the space environment. ${ }^{1,2}$ Microgravity in the space is not appropriate to humans, and can affect the health of space man..$^{3-5}$ The bone is one of the affected organs. ${ }^{6}$ So, the purpose of this mini review was to discuss about the effects of microgravity on bone.

\section{Discussion}

The human body is structured to sustain forces involved in the Earth. Under adverse conditions, for example microgravity, the body can undergo physiological changes. Bone loss is the most recognized phenomenon related to microgravity. ${ }^{7}$ Bone is an active organ that maintains balance of bone formation and bone resorption; in normal conditions. $^{8}$ It is considered one anisotropic material, plastic and resistant. Bone is an active organ having constant activity during the life, with an increasing in bone resorption in late life. Bone remodeling balance relates a variety of mechanisms, internal and external factors can affect this regulation causing bone disease. ${ }^{9}$ For example osteoporosis is a disease characterized by low bone mineral density, high bone porosity and low bone strength. Bone in this condition is weakened and fracture risk increases. ${ }^{9}$ Old age people could develop osteoporosis for changes of hormonal levels in post menopause. Furthermore, osteoporosis can be originated by other conditions in which bone is deprived of mechanical stimulus. This condition characterizes disuse osteoporosis and can be irreversible Spinal cord injury, prolonged bed rest and astronauts submitted to microgravity can develop disuse osteoporosis. ${ }^{10,11}$

Astronauts and cosmonauts in space are confined to an environment with microgravity. Thus, the effects of load on bone are absent, which mimic disuse conditions. ${ }^{5,6,12} \mathrm{An}$ important characteristic of bone is the capacity to respond to mechanical stimulus that has been submitted by mechanotransduction. ${ }^{13}$ So, to maintain balance formation and resorption the bone need to be stimulated. Daily activities, body weight under positive gravity and muscle contraction provide mechanical stimulus for bone. ${ }^{14}$ Skeletal metabolism responds to physical activity by alteration on bone mass and bone architecture. ${ }^{13}$ Bones of astronauts are not recruited in this way during space flights, thus bone mineral density is reduced..$^{15}$ The rate of $1-2 \%$ of bone mineral loss per month in at least one skeletal site was registered during space flights. ${ }^{6}$ In crewmembers of Apollo 15 was reported over time 5 and $6 \%$ of bone density loss. This loss can be influenced by the length of the space flight mission. ${ }^{6}$ In the study of Collet et al., ${ }^{16}$ bone mineral density was evaluated in two cosmonauts. After 1 month of exposure to microgravity a loss in trabecular bone mass was observed, being more marked after 6 months. ${ }^{16}$

In space flights, an increase in calcium excretion has been observed. Indeed, unbalance of parathyroid hormone (PTH) levels was reported in spinal cord injured patients. ${ }^{17}$ These evidences seem to explain bone mineral density loss. The central energy control influences PTH levels on calcium and phosphate metabolism, which can be involved on bone loss..$^{18}$ Other evidence is that trabecular bone loss has been more intense than cortical bone loss. ${ }^{16}$ In Skylab IV bone loss in calcaneus was observed, but no difference was found in radius and ulna, confirming trabecular bone is more susceptible to bone loss. ${ }^{6}$ Trabecular bone has faster response to change in microenvironment and can be inferred that weight bearing bones are more affected by microgravity. Short periods of immobilization have been demonstrated significative bone loss. ${ }^{11}$ During static position bone of the hindlimb is forced by gravity to support body weight. Indeed, dynamic loading generated by muscles contraction and reaction forces stimulate bone of hindlimbs. Dynamics force can have stronger effects on bone mass than static forces. ${ }^{19}$ In parallel with bone loss occur muscle mass loss mainly of loaded muscles. ${ }^{20}$ Confirming that there is a strong relation between muscle activity and bone metabolism. If bone is not required it has not remodeling balance to maintain its structural integrity. For this reason, disuse osteoporosis is a local phenomenon affecting generally lower limbs.

Effects of microgravity also are observed in the vascular system. Physical exercise increase bone marrow hydrostatic pressure, which is osteogenic factor. In the space, bone blood flow is changed by alterations in pressure and consequently the response of osteoprogenitor cells in the bone marrow could be affected. In addition, blood flow could do not offer oxygen and nutrients in an adequate way to support development of bone cells. ${ }^{21}$ At same time, 
the energy metabolism suffers an unbalance and affect other hormone actions as adiponectin and insulin. As the body did not expend energy it is accumulating in body fat. High adipocyte content in bone marrow was found in patients with spinal cord injury ${ }^{22}$ and submitted to long term bed rest. ${ }^{23}$ This may be an evidence that immobilization causes an unfavorable environment to osteoblasts formation. Osteoblasts and adipocytes have a common progenitor, so mesenchymal stem cells could be preferentially differentiated into adipocytes; disfavoring osteoblasts. ${ }^{22}$ Spinal cord injured patients have been developed insulin resistance. ${ }^{24}$ Insulin resistance is related to high adipocyte and has a partly action on bone matrix degradation. Pathophysiology of disuse osteoporosis by microgravity is not completely understood. But, based on evidences is possible inferred that a series of physiological mechanisms seem be associated causing bone matrix, structural and functional depletion in weightless condition. Difficulty in acquiring data due lower number spaceflight missions limits searches to evaluate the influence of microgravity in bone loss. ${ }^{25}$ Although both have common consequences, bed rest immobilization is not perfect to mimic microgravity, because astronauts were submitted to reduced loading but not completely inactive. For this purpose, animal models have been proposed to give answers about this field. Experimental models are cost-effective and succeed evidences about how body system is affected by weightlessness. ${ }^{26}$

The hindlimb unloading method is applied in mouse and rats in which animals are maintained in tail suspension reproducing disuse of the hindlimbs that not touch the ground. ${ }^{27}$ Animals have freedom to eat, drink and move in the cage with the forelimbs. The angle formed between the torso of the animal and the floor of the cage must be $30^{\circ}$ to maintain adequate loading in the forelimbs and traction on the tail. Moreover, this position allows cephalad fluid shift and maintain adapted load on vertebrae with minimal evidence of stress. ${ }^{28}$ However, tail suspension cause unload only in the hindlimb whereas spaceflight cause entire body unload, tail suspension model has showed similar responses in diverse body system as pulmonary, intestine, endocrine and musculoskeletal. So, this model was compatible to spaceflight to study bone alterations. ${ }^{29}$ The animals submitted to tail suspension present reduction in bone formation, altered calcium balance with high calcium excretion and reduction in bone strength, similarity is found in space flight studies..$^{25}$ Weight bearing bones are more affected by microgravity than no weight bearing bones, so tail suspension seems a reproducible method to study the effects of unload in bone. Tail suspension has been reduced bone mineral density and mechanical properties of long bones. ${ }^{30}$ Depletion in bone due tail suspension can be explained by inhibition of bone formation and enhanced bone resorption. ${ }^{26}$ Bone mineral density and strength decreased in femurs of suspended rats. ${ }^{31}$ A period of suspension for 1 week is able to reduce relative trabecular bone (BV/TV), mineralized trabecular bone surface (MS/BS) and bone formation rate at the same time that increase osteoclast surface (Oc.S/BS) and number (Oc.N/ BS) in mice. ${ }^{32}$ In vitro study confirms that skeletal unloading inhibits proliferation and differentiation of osteoprogenitor cells. ${ }^{22}$ Osteocytes also can be affected by unloading stimulus in a bioreactor, with a high expression of inhibitors of bone formation (sclerotin) and stimulators of bone resorption (RANKL). ${ }^{33}$ Studies in vitro provide insights on news therapies targeting directly cells in bone.

To demonstrate disuse osteoporosis other animal models are used as neurectomy, tenotomy and plaster cast. ${ }^{26}$ Sciatic neurectomy is a model used to preclude movements of hindlimbs. ${ }^{34}$ Tenotomy consist in a cut of anterior, lateral and medial tendons of femur and tibia causing hind limb immobilization. ${ }^{35}$ A method using plaster cast cause a disuse in which 10 days are enough to cause bone loss in rats. ${ }^{36}$ However, the phenotype of tail suspension seems more appropriate to mimic space flights. Tail suspension causes an unload in hindlimbs and not a total immobilization. Indeed, it is possible to mimic circulation of fluids, to minimize stress and to maintain neuro system intact, once bone cells have receptors for neuromediators. The negative influence of tail suspension in bone have not been related to abnormally stress, confirmed by plasmatic corticosterone levels found not elevated in unloading animal model ${ }^{37}$ The model is ethical and equivalent to bone alterations caused by spaceflights. A variety of analysis not applicable in human can be reproducible in an animal model, with significative relevance to improve techniques and advances in the prevention and treatments of bone loss. Osteoporosis can be irreversible. When human back to Earth their bone can be not able to return normal activities with load and bone weaker can be conducted to fractures or other musculoskeletal injuries. ${ }^{11}$ After ninety days of Skylab IV calcaneus was evaluated and bone mineral density was not restored. ${ }^{38}$ Experimental studies showed reloading after a period of 1 week suspension can restore bone weakness, but after 2 weeks bone restore was not satisfactory. ${ }^{32}$ Also, same time short-duration of loading did not prevent deleterious effects on bone of disuse. ${ }^{39}$

Exercise has been an effective measure to various physiological systems to avoid negative effects in a microgravity environment, but no effective effects were observed in bone loss. ${ }^{6,11}$ Muscle activity is an important stimulus to bone remodeling nevertheless in absence of load seems alone not able to prevent bone loss. In an attempt to avoid bone attenuation countermeasures were considered, as using of pharmaceutical and anabolic agents. ${ }^{6}$ Bisphosphonates, testosterone and $\mathrm{K} 2$ vitamin associated with physical exercise can be an alternative to minimize side effects of microgravity. ${ }^{15}$ Low intensity pulsed ultrasound demonstrated to be a non-invasive countermeasure to mitigate the adverse effects of disuse osteoporosis. ${ }^{40}$ Associated measures and equipment might be a solution to prevent bone loss. So, animal models offer a possibility to study new interventions. The study of Lau et al., ${ }^{41}$ suggests that electrical stimulation at the dorsal root ganglion of rats can effectively prevent bone loss from unloading hindlimb and that model animals are useful alternative to investigate new modalities of intervention. Other experiment supports the notion that endoplasmic reticulum stress plays a key role in the pathogenesis of disuse osteoporosis, and salubrinal might be a therapeutic agent. ${ }^{42}$ The rehabilitation in animals is faster, but disuse can cause non-regressive alterations in humans. For this reason, preventive interventions are desirable.

\section{Conclusion}

Experimental studies using hindlimb unloading method shown bone loss in animal models and have been effective in simulating the effects of microgravity. Therapies to reverse osteopenia and osteoporosis are being investigated. These therapies include physical and chemical agents. Experimental studies in animals are fundamental to understanding the effects and mechanisms of microgravity action. From this knowledge, it will be possible to develop prevention and treatment strategies for bone loss in astronauts and cosmonauts.

\section{Acknowledgements}

The authors gratefully acknowledge the scholarship from the Coordination for the Improvement of Higher Education Personnel. 


\section{Conflict of interest}

All authors have no conflicts of interest.

\section{References}

1. Zwart SR, Gibson CR, Gregory JF, et al. Astronaut ophthalmic syndrome. FASEB J. 2017;31(9):3746-3756.

2. Fernandez-Gonzalo R, Baatout S, Moreels M. Impact of Particle Irradiation on the Immune System: From the Clinic to Mars. Front Immunol. 2017;8:177.

3. Chang DG, Healey RM, Snyder AJ, et al. Lumbar Spine Paraspinal Muscle and Intervertebral Disc Height Changes in Astronauts After Long-Duration Spaceflight on the International Space Station. Spine. 2016;41(24):1917-1924.

4. Boerma M, Nelson GA, Sridharan V, et al. Space radiation and cardiovascular disease risk. World J Cardiol. 2015;7(12):882-888.

5. Vico L, Van Rietbergen B, Vilayphiou N, et al. Cortical and trabecular bone microstructure did not recover at weight-bearing skeletal sites and progressively deteriorated at non-weight-bearing sites during the year following International Space Station missions. J Bone Miner Res. 2017;32(10):2010-2021.

6. LeBlanc AD, Spector ER, Evans HJ, et al. Skeletal responses to space flight and the bed rest analog: a review. J Musculoskelet Neuronal Interact. 2007;7(1):33-47.

7. Bloomfield SA, Martinez DA, Boudreaux RD, et al. Microgravity Stress: Bone and Connective Tissue. Compr Physiol. 2016;6(2):645-686.

8. Robling AG, Castillo AB, Turner CH. Biomechanical and Molecular Regulation of Bone Remodeling. Annu Rev Biomed Eng. 2006;8:455498.

9. Paiva AG, Yanagihara GR, Macedo AP, et al. Analysis of fracture healing in osteopenic bone caused by disuse: experimental study. Braz J Med Biol Res. 2016;49(3):1-7.

10. Minaire P, Neunier P, Edouard C, et al. Quantitative histological data on disuse osteoporosis: comparison with biological data. Calcif Tissue Res. 1974; 17(1):57-73.

11. Shimano MM, Volpon JB. Biomechanics and structural adaptations of the rat femur after hindlimb suspension and treadmill running. Braz J Med Biol Res. 2009;42(4):330-338.

12. Cappellesso R, Nicole L, Guido A. Spaceflight osteoporosis: current state and future perspective. Endocr Regul. 2015;49(4):231-239.

13. Ducan RL, Tumer $\mathrm{CH}$. Mechanotransduction and the functional response of bone to mechanical strain. Calcif Tissue Int. 1995;57(5):344-358.

14. Alexandre C, Vico L. Pathophysiology of bone loss in disuse osteoporosis. Joint Bone Spine. 2011;78(6):572-576.

15. Iwamoto J, Takeda T, Sato Y. Interventions to prevent bone loss in astronauts during space flight. Keio J Med. 2005;54(2):55-59.

16. Collet P, Uebelhart D, Vico L, et al. Effects of 1- and 6-month spaceflight on bone mass and biochemistry in two humans. Bone. 1997;20(6):547551.

17. Jiang S, Dai L, Jiang L. Osteoporosis after spinal cord injury. Osteoporos Int. 2006;17(2):180-192.

18. Carbuhn AF, Fernandez TE, Bragg AF, et al. Sport and training influence bone and body composition in women collegiate athletes. J Strength Cond Res. 2010;24(7):1710-1717.
19. Tumer $\mathrm{CH}$, Burr DB. Basic biomechanical measurements of bone: a tutorial. Bone. 1993;14(4):595-608.

20. Ducher G, Courteix D, Même, et al. Bone geometry in response to long-term tennis playing and its relationship with muscle volume: a quantitative magnetic resonance imaging study in tennis players. Bone. 2005;37(4):457-466.

21. Nagatomi J, Arulanandam BP, Metzger DW, et al. Cyclic pressure affects osteoblast functions pertinent to osteogenesis. Ann Biomed Eng. 2003;31(8):917-923.

22. Kostenuik PJ, Halloran BP, Morey-holton ER, et al. Skeletal unloading inhibits the in vitro proliferation and differentiation of rat osteoprogenitor cells. Am J Physiol. 1997;273(6 Pt 1):E1133-1139.

23. Trudel G, Payne M, Madler B, et al. Bone marrow fat accumulation after 60 days of bed rest persisted 1 year after activities were resumed along with hemopoietic stimulation: the Women International Space Simulation for Exploration study. J Appl Physiol. 2009;107(2):540-548.

24. Gorgey AS, Mather KJ, Gater DR. Central adiposity associations to carbohydrate and lipid metabolism in individuals with complete motor spinal cord injury. Metabolism. 2011;60(6):843-851.

25. Bikle DD, Halloran BP, Morey-Holton E. Spaceflight and the skeleton: lessons for the earthbound. Gravit Space Biol Bull. 1997;10(2):119-135.

26. Komori T. Animal models for osteoporosis. Eur J Pharmacol. 2015;759:287-294.

27. Morey-Holton ER, Globus RK. Hindlimb unloading rodent model: technical aspects. J Appl Physiol. 2002;92(4):1367-1377.

28. Globus RK, Bikle DD, Morey-Holton E. The Temporal Response of Bone to Unloading. Endocrinology. 1986;118(2):733-742.

29. Morey-Holton ER, Globus RK. Hindlimb unloading of growing rats: a model for predicting skeletal changes during space flight. Bone. 1998;22(5 Suppl):83S-88S.

30. Yanagihara GR, Paiva AG, Gasparini GA, et al. High-impact exercise in rats prior to and during suspension can prevent bone loss. Braz J Med Biol Res. 2016;49(3):1-8.

31. Falcai MJ, Zamarioli A, Okubo R, et al. The osteogenic effects of swimming, jumping, and vibration on the protection of bone quality from disuse bone loss. Scand J Med Sci Sport. 2015;25(3):390-397.

32. Sakai A, Nakamura T. Changes in trabecular bone turnover and bone marrow cel development in tail-suspended mice. J Musculoskelet Neuronal Interact. 2001;1(4):387-392.

33. Spatz JM, Wein MN, Gooi JH, et al. The Wnt inhibitor sclerostin is upregulated by mechanical unloading in osteocytes in vitro. $J$ Biol Chem. 2015;290(27):16744-16758.

34. Brighton CT, Tadduni GT, Goll SR, et al. Treatment of denervation/ disuse osteoporosis in the rat with a capacitively coupled electrical signal: effects on bone formation and bone resorption. J Orthop Res. 1988;6(5):676-684.

35. Shaker JL, Fallon MD, Goldfarb S, et al. WR-2721 reduces bone loss after hindlimb tenotomy in rats. J Bone Miner Res. 1989;4(6):885-890.

36. Hott M, Deloffre P, Tsouderos Y, et al. S12911-2 reduces bone loss induced by short-term immobilization in rats. Bone. 2003;33(1):115123.

37. Halloran BP, Bikle DD, Cone CM, et al. Glucocorticoids and Inhibition of Bone Formation Induced by Skeletal Unloading. Am J Physiol. 1988;255(6 Pt 1):E875-879. 
38. LeBlanc A, Schneider V. Can the adult skeleton recover lost bone? Exp Gerontol. 1991;26(2-3):189-201.

39. Leung KS, Li YH, Liu Y, et al. Effect of daily short-duration weightbearing on disuse-induced deterioration of musculoskeletal system. $J$ Musculoskelet Neuronal Interact. 2015;15(2):207-214.

40. Uddin SMZ, Qin YX. Dynamic acoustic radiation force retains bone structural and mechanical integrity in a functional disuse osteopenia model. Bone. 2015;75:8-17.
41. Lau RY-C, Qian X, Po KT, et al. Response of rat tibia to prolonged unloading under the influence of electrical stimulation at the dorsal root ganglion. Neuromodulation. 2017;20(3):284-289.

42. Li J, Yang S, Li X, et al. Role of endoplasmic reticulum stress in disuse osteoporosis. Bone. 2017;97:2-14. 